\title{
Лекарственно-индуцированный остеопороз
}

\author{
О.Д. Остроумова ${ }^{1,2}$, И.В. Голобородова
}

ФФГБОУ ДПО

“Российская медицинская академия непрерывного профессионального образования" Минздрава РФ, ${ }^{2}$ ФГАОУ ВО “Первый Московский государственный медицинский университет имени И.М. Сеченова" Минздрава РФ (Сеченовский

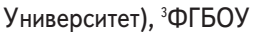
ВО “Московский государственный медико-стоматологический университет им. А.И. Евдокимова", Москва, Россия

Для корреспонденции: О.Д. Остроумова, РМАНПО Минздрава РФ, Москва, 125993, г. Москва, ул. Барри кад ная, д. 2/1, стр. 1 , ostroumova.olga@mail.ru;

Для цитирования: Остроумова О.Д., Голо бородова И.В. Лекар ственно-индуцированный остеопороз. Клин фармакол тер 2020;29(3):73-79 [Ostroumova OD, Golobo rodova IV. Drug-induced osteoporosis. Kliniches kaya farmakologiya i terapiya $=$ Clin Pharmacol Ther 2020;29(3):73-79 (In Russ.)]. DOI 10.32756/08695490-2020-3-73-79.
Остеопороз характеризуется снижением костной массы, нарушением микроархитектоники костной ткани и возникновением переломов при минимальной травме. Выделяют первичный, ассоциированный со старением и снижением фрункции половых желез, и вторичный остеопороз. Одной из причин вторичного остеопороза являются лекарственные средства (ЛС), в том числе гормональные (системные глюкокортикостероиды, ингибиторы ароматазы, депо-медроксипрогестерон, агонисты гонадотропин-рилизинг гормона, левотироксин), антисекреторные (ингибиторы протонной помпы, блокаторы $\mathrm{H}_{2}$-гистаминовых рецепторов), психотропные (противоэпилептические препараты, антидепрессанты), сахароснижающие (тиазолидиндионы) средства, а также ингибиторы кальциневрина, антиретровирусные препараты, антикоагулянты, некоторые химиотерапевтические препараты, петлевые диуретики. ЛС способны приводить к потере костной массы, вмешиваясь непосредственно в различные этапы процесса ремоделирования (восстановления, перестройки) кости, а также опосредованно, влияя на уровни половых гормонов и кальциево-фросфорный обмен. К фракторам риска лекарственно-индуцированного остеопороза относят низкое потребление кальция, употребление алкоголя, курение, недостаточную физическую активность, а также пожилой возраст, женский пол, падения и/или переломы в анамнезе, сниженные массу тела или индекс массы тела, постменопаузальный статус, отягощенный семейный анамнез в отношении переломов. Профилактика лекарственно-индуцированного вторичного остеопороза предполагает отказ от применения ЛС, вызывающего это осложнение, или его замену на другой препарат, не дающий такого побочного эфффекта. Если полностью отказаться от приема препарата невозможно, для сведения к минимуму риска развития остеопороза необходимо снизить дозу препарата, уменьшить продолжительность терапии или назначить коррекционную терапию.

Ключевые слова. Остеопороз, лекарстВенно-индуцироВанный остеопороз, потеря костной массы, остеопоретический перелом, лекарстВенно-индуцированная потеря костной массы, нежелательные лекарстВенные реакции.

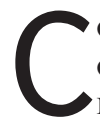
огласно определению экспертов ВОЗ, остеопороз (ОП) представляет собой метаболическое заболевание скелета, характеризующееся снижением костной массы, нарушением микроархитектоники костной ткани и, как следствие, переломами, возникающими при минимальной травме, например, при падении с высоты собственного роста, неловком движении, кашле, чихании (т.н. низкоэнергетические переломы), или без видимого травматического вмешательства [1].

ОП является одним из самых распространенных заболеваний и значимых медикосоциальных проблем. Так, более 200 миллионов человек во всем мире страдают ОП, при этом на страны Северной и Южной Америки, Европы и Японии приходится более 75 млн больных [2,3]. Ежегодно в мире ОП вызывает более 8,9 миллионов переломов, из которых на страны Северной и Южной Америки и Европы приходится более 4,5 миллионов [2]. Данные Между на родного фонда остеопороза (International Osteoporosis Foundation, IOF) [4] свидетельствуют о том, что каждые три секунды в мире происходит один остеопоретический перелом, а, начиная с 50-летнего возраста, каждая третья женщина и каждый пятый мужчина в течение оставшейся жизни перенесут хотя бы один остеопоретический перелом. Предполагается, что пожизненный риск возникновения перелома запястья, бедра или позвоночника в развитых странах мира составляет около $30-40 \%$, приближаясь к показателям риска сердечно-сосудистых 
осложнений [2].

В Российской Федерации ОП страдают около 14 млн человек (10\% населения страны) [1]. В возрасте $\geq 50$ лет ОП выявляют у $34 \%$ женщин и $27 \%$ мужчин, а приз наки остеопении, характеризующейся снижением минеральной плотности костей (МПК) и также ассоциированной с развитием низкоэнергетических переломов, определяются более чем у $40 \%$ мужчин и женщин [1]. Одномоментное эпидемиологическое исследование среди городского населения России в возрасте 50 лет и старше показало, что 24\% женщин и $13 \%$ мужчин ранее уже перенесли по крайней мере один низкоэнергетический перелом [1]. Известно, что у женщин развитие ОП и переломов отмечается чаще, чем у мужчин. Так, по сравнению с мужчинами у женщин в возрасте $\geq 50$ лет в 7 раз чаще наблюдались переломы лучевой кости, в 2-3 раза чаще - переломы шейки бедренной кости, что обусловлено быстрой потерей костной массы у женщин в климактерическом периоде (более $3 \%$ в год и до $40 \%$ в течение всей оставшейся жизни).

Кроме того, частота ОП увеличивается с возрастом [1]. Учитывая современные демографические тенденции, характеризующиеся увеличением продолжительности жизни населения и числа пожилых людей, ожидается, что к 2050 г. частота остеопоротических переломов увеличится в 2,4 раза [1].

Однако актуальность проблемы ОП связана не только со значительной распространенностью и высоким риском возникновения остеопоретических переломов. ОП и его социальные последствия - снижение качества жизни, высокий уровень инвалидизации и даже смерти от осложнений, находятся в фокусе внимания медицинской общественности. По данным И.И. Дедова и соавт. [5], в течение первого года после перелома бедра смертность составляет $12-40 \%$, при этом наиболее высокие показатели смертности регистрируются в первые 6 мес после перелома [1]. Среди выживших после перелома бедра каждый третий утрачивает способность к самообслуживанию и нуждается в длительном постоянном уходе [6]. В странах Северной и Южной Аме рики и Европе остеопоретические переломы составляют около 2,8 миллионов лет жизни с поправкой на инвалидность, что превышает аналогичный показатель для гипертонический болезни и ревматоидного артрита [3].

Важной стороной проблемы ОП являются и экономические последствия заболевания. В странах Евросою за в 2010 г. на лечение остеопоретических переломов было потрачено 37 млрд евро. Ожидается, что к 2025 году эти затраты возрастут на 25\% [3]. В России усредненная стоимость одного года лечения ОП, осложненного переломом, составляет 61151 руб. [1]. Пересчет на население России ( $\geq 50$ лет) свидетельствует о том, что прямые медицинские затраты на лечение низкоэнергетических переломов за один год могут достигать 25 млрд рублей [1].

Высокая медико-социальная и экономическая зна чимость проблемы ОП стимулирует продолжение изу чения эпидемиологических, медицинских, социальных, экономических аспектов проблемы. Во многих странах созданы и успешно функционируют профессиональные ассоциации и службы (IOF, Global Alliance for Musculoskeletal Health и др.), созданные с целью снижения бремени заболевания и осуществляющие постоянный поиск и продвижение экономически эффективных методов профилактики и лечения больных с заболеваниями опорно-двигательного аппарата, включая ОП. Одно из направлений деятельности этих организаций включает оценку лекарственных средств (ЛС), которые могут вызвать развитие ОП.

Уменьшение костной массы, не связанное с другими хроническими заболеваниями, называется первичным ОП, обусловленным старением и снижением функции половых желез (постменопаузальный период) [1]. Вторичный ОП рассматривается как заболевание, развивающееся вследствие воздействия любых факторов, отличных от старения и постменопаузального периода, таких как серьезные сопутствующие соматические заболевания или прием лекарственных препаратов (лекарственно-индуцированный остеопороз). вторичным ОП потеря костной массы превышают таковую при старении и менопаузе, т.е. первичном ОП Именно поэтому особенно важно обеспечить своевременную идентификацию и адекватную терапию пациентов со вторичным ОП.

Развитие лекарственно-индуцированного ОП ассоциировано с приемом ряда ЛС [1,3,7-10]: гормональных (системные глюкокортикостероиды [ГКС], ингибиторы ароматазы, депо-медроксипрогестерон, агонисты гонадотропин-рилизинг гормона [ГнРГ], левотироксин), антисекреторных (ингибиторы протонной помпы [ИПП], блокаторы $\mathrm{H}_{2}$-гистаминовых рецепторов), психотропных (противоэпилептические препараты, антидепрессанты), сахароснижающих ЛС (тиазолидиндионы), а также ингибиторов кальциневрина, антиретровирусных препаратов, антикоагулянтов, некоторых химиотерапевтических препаратов, петлевых диуретиков (табл. 1).

\section{Эпидемиология и механизмы развития лекарственно-индуцированного остеопороза}

Вторичный ОП встречается достаточно часто, однако точные данные о его распространенности отсутствуют. Имеются данные о том, что более двух третей мужчин, а также более половины женщин в пременопаузе и приблизительно одна треть женщин в постменопаузе с ОП имеют то или иное патологическое состояние и/или принимают ЛС, способствующие потере костной массы [11]. При этом определить частоту развития лекарственно-индуцированного ОП трудно, так как ЛС обычно способствуют увеличению риска ОП, а не являются его единственной причиной [3].

Костная ткань в течение жизни человека находится в состоянии постоянного изменения. Процесс, посредством которого кость подвергается восстановлению и перестройке, называется ремоделированием костной ткани. В этом процессе участвуют три основные группы 
ТАБЛИЦА 1. Лекарственные средства, применение которых ассоциировано с остеопорозом и переломами [3,7-10]

\begin{tabular}{|c|c|c|c|}
\hline Препараты & Частота и/или риск & Предполагаемые механизмы & $\begin{array}{l}\text { Доказан- } \\
\text { ность }\end{array}$ \\
\hline $\begin{array}{l}\text { Противоэпилептические } \\
\text { ЛС }\end{array}$ & $\begin{array}{l}\text { МПК: потеря костной массы 0,35-1,8\%; Переломы: ОР } \\
2,18 \text { (95\% ДИ: } 1,94-2,45)\end{array}$ & $\begin{array}{l}\text { Выраженная недостаточность витамина } \\
\text { D, снижение усвоения кальция }\end{array}$ & B \\
\hline $\begin{array}{l}\text { Антиретровирусная } \\
\text { терапия }\end{array}$ & $\begin{array}{l}\text { МПК: потеря костной массы 1-6\%; пациенты с ОП, } \\
\text { получающие антиретровирусную терапию - ОШ 2,38 } \\
\text { (95\% ДИ: } 1,20-4,75) \text {; пациенты с ОП, получающие инги- } \\
\text { биторы протеаз - ОШ 1,57 (95\% ДИ: 1,05-2,34); } \\
\text { Переломы: нет данных }\end{array}$ & $\begin{array}{l}\text { Увеличение активности остеокластов, } \\
\text { снижение экспрессии OPG }\end{array}$ & B \\
\hline Ингибиторы ароматазы & $\begin{array}{l}\text { МПК: потеря костной массы 2,5-5\% в позвоночнике, } \\
1,5-5 \% \text { в бедре (в среднем } 2 \% \text { в год); } \\
\text { Переломы: 7,1\% для анастрозола; } 5,7 \% \text { для летрозола }\end{array}$ & $\begin{array}{l}\text { Снижение функциональных } \\
\text { концентраций эстрогена вследствие } \\
\text { подавления ароматизации андрогенов }\end{array}$ & B \\
\hline Тиазолидиндионы & $\begin{array}{l}\text { МПК: потеря костной массы до 0,6-1,2\% в год; } \\
\text { Переломы: до 9\%, у женщин ОШ 1,94 (95\% ДИ:1,60-2,35; } \\
\text { р<0,001), у мужчин ОШ 1,02 (95\% ДИ: } 0,83-1,27 ; \mathrm{p}=0,83 \text { ) }\end{array}$ & $\begin{array}{l}\text { Снижение дифференцировки и функции } \\
\text { остеобластов }\end{array}$ & B \\
\hline Циклоспорин & Нет данных & $\begin{array}{l}\text { Стимуляция ремоделирования костной } \\
\text { ткани, влияние на метаболизм остеокаль- } \\
\text { цина и витамина D }\end{array}$ & $\mathrm{C}$ \\
\hline $\begin{array}{l}\text { Депо-медроксипроге - } \\
\text { стерон }\end{array}$ & $\begin{array}{l}\text { МПК: потеря костной массы 2-8\%; } \\
\text { Переломы: частота по сравнению с не использующими } \\
1,41 \text { (95\% ДИ: } 1,35-1,47)\end{array}$ & $\begin{array}{l}\text { Подавление гипоталамо-гипофизарно- } \\
\text { яичниковой оси со снижением уровня } \\
\text { эстрогена }\end{array}$ & B \\
\hline $\begin{array}{l}\text { Агонисты гонадотропин- } \\
\text { рилизинг гормона }\end{array}$ & $\begin{array}{l}\text { МПК: потеря костной ткани бедра 6-7\%; трабекулярной } \\
\text { кости - 5-10\%; } \\
\text { Перелом: позвонка - ОР 1,45 (95\% ДИ: 1,19-1,75); } \\
\text { бедренной кости - ОР 1,3 (95\% ДИ: 1,1-1,53) }\end{array}$ & $\begin{array}{l}\text { Подавление выработки } \\
\text { тестостерона }\end{array}$ & B \\
\hline Диуретики (фуросемид) & $\begin{array}{l}\text { МПК: увеличение потери костной массы на } 0,3 \% \text { в год; } \\
\text { Переломы: ОР 3,9 (95\% ДИ: } 1,5-10,4)\end{array}$ & $\begin{array}{l}\text { Стимуляция почечной экскреции } \\
\text { кальция }\end{array}$ & B \\
\hline $\begin{array}{l}\text { Метотрексат (высокие } \\
\text { дозы) }\end{array}$ & $\begin{array}{l}\text { МПК: потеря костной массы 0,2-1,6\%; } \\
\text { Переломы: } 12-45 \%\end{array}$ & $\begin{array}{l}\text { Стимуляция апоптоза остеоцитов, } \\
\text { увеличение числа остеокластов }\end{array}$ & $\mathrm{C}$ \\
\hline Левотироксин & $\begin{array}{l}\text { МПК: потеря костной массы 10-12\%; } \\
\text { Переломы: ОР 1,56 (95\% ДИ: 1,03-2,37), наиболее } \\
\text { высокий при дозах >150 мкг/сут }\end{array}$ & $\begin{array}{l}\text { Повышенная активация остеокластов и } \\
\text { RANKL }\end{array}$ & B \\
\hline $\begin{array}{l}\text { Ингибиторы протонной } \\
\text { помпы }\end{array}$ & $\begin{array}{l}\text { МПК: нет данных; } \\
\text { Переломы: ОР 1,28 (95\% ДИ: 1,13-1,44) }\end{array}$ & Снижение абсорбции кальция & B \\
\hline $\begin{array}{l}\text { Ингибиторы обратного } \\
\text { захвата серотонина }\end{array}$ & $\begin{array}{l}\text { МПК: потеря костной массы 4,4-6,2\%; } \\
\text { Переломы: ОР 1,61 (95\% ДИ: 1,49-1,74) }\end{array}$ & $\begin{array}{l}\text { Возможно ингибирование системы } \\
\text { транспорта серотонина в остеобластах } \\
\text { со снижением их активности }\end{array}$ & B \\
\hline Системные ГКС & $\begin{array}{l}\text { МПК: показатели ниже, чем у лиц аналогичного возраста } \\
\text { и пола; } \\
\text { Переломы: 30-50\%; ОР 1,25 (95\% ДИ: 1,07-1,45), } \\
\text { перелом бедра ОР 1,61 (95\% ДИ: 1,18-2,20) }\end{array}$ & $\begin{array}{l}\text { Угнетающее действие на остеобласты, } \\
\text { усиленный апоптоз остеоцитов, увеличе- } \\
\text { ние экспрессии RANKL и уменьшение } \\
\text { экспрессии OPG, нижение выработки } \\
\text { половых гормонов, уменьшение всасыва- } \\
\text { ния кальция в кишечнике, увеличение } \\
\text { экскреции кальция с мочой. }\end{array}$ & A \\
\hline $\begin{array}{l}\text { Нефракционированный } \\
\text { гепарин и низкомолеку- } \\
\text { лярный гепарин }\end{array}$ & $\begin{array}{l}\text { МПК: потеря до } 30 \% \text { костной массы; } \\
\text { Переломы: 2,2-3,6\% }\end{array}$ & $\begin{array}{l}\text { Снижение экспрессии ОРG, подавление } \\
\text { дифференцировки и функции остеобла- } \\
\text { стов }\end{array}$ & B \\
\hline Варфарин & $\begin{array}{l}\text { МПК: потеря до 9-10,4\% костной массы; } \\
\text { Переломы: позвонка - SIR 2,4 (95\% ДИ:1,6-3,4) через } \\
3 \text { мес; 3,6 (95\% ДИ:2,5-4,9) от } 3 \text { до 12 мес и 5,3 (95\% } \\
\text { ДИ:3,4-8,0) } 12 \text { и более мес; ребер - 1,6 (95\% ДИ:0,9-2,7) } \\
\text { через 3 мес; 1,6 (95\% ДИ:0,9-2,6) от } 3 \text { до } 12 \text { мес; } \\
\text { 3,4 (95\% ДИ:1,8-5,7) через 12 мес. }\end{array}$ & $\begin{array}{l}\text { Возможно ингибирование } \gamma \text {-карбоксили- } \\
\text { рования со снижением кальций-связы- } \\
\text { вающих свойств остеокальцина }\end{array}$ & $\mathrm{C}$ \\
\hline HOAK & $\begin{array}{l}\text { МПК: ОР 0,82 ( } 95 \% \text { ДИ: 0,68-0,97) по сравнению } \\
\text { с варфарином; } \\
\text { Переломы: ОР 0,84 (95\% ДИ: 0,77-0,93) } \\
\text { по сравнению с варфарином. }\end{array}$ & Нет данных & B \\
\hline
\end{tabular}

Примечание. ОР - относительный риск или отношение рисков, ОШ - отношение шансов, ДИ - доверительный интервал, ГКС - глюкокортикостероиды; МПК- минеральная плотность кости, HOAK - новые оральные антикоагулянты, ЛС - лекарственное средство, RANKL - receptor activator of nuclear factor kappa-B ligand, OPG - osteoprotegerin, SIR - стандартизованное отношение заболеваемости. *Уровни доказанности [3]: А - данные одного и более рандомизированных исследований; В - данных нерандомизированных клинических исследований, проспективных обсервационных исследований, когортных исследований, ретроспективных исследований типа случай-контроль, мета-анализов и/или постмаркетинговых наблюдательных исследований; С - описание случая или серии случаев 
клеток: остеобласты, остеоциты и остеокласты. Остеобласты происходят от предшественника мезенхимальных стволовых клеток и отвечают за синтез органического костного матрикса (остеоида) и минерализацию кости. Остеоциты - зрелые клетки кости, которые представляют собой удаленные остеобласты, "замурованные" в кости. Они являются клетками связи, помогающими координировать цикл ремоделирования в определенном месте. Остеокласты происходят от гематопоэтических предшественников линии моноцитовмакрофагов и ответственны за резорбцию кости [3].

Процесс ремоделирования начинается, когда лиганд рецептора активатора ядерного транскрипционного фактора каппа B (receptor activator of nuclear factor kappa-B ligand, RANKL), секретирующийся клеткамипредшественницами остеобластов, связывается со своим рецептором (receptor activator of nuclear factor kappa-B, RANK) на поверхности клеток-предшественниц остеокластов. Результатом взаимодействия является стимуляция остеокластогенеза с дифференцировкой и активацией зрелых остеокластов, которые осуществляют резорбцию кости посредством деградации белкового матрикса и деминерализации. После завершения резорбции кости в этом месте высвобождаются цитокины и факторы роста, которые участвуют в хемотаксисе, пролиферации и дифференцировке остеобластов [3].

Остеопротегерин (osteoprotegerin, OPG) - растворимый белок, также являющийся важной составляющей системы RANK и секретирующийся клетками-предшественницами остеобластов. OPG, способный связывать RANKL, выполняет функцию "рецепторной ловушки" для данного лиганда. Взаимодействие OPG и RANKL приводит к ингибированию дифференцировки и активации остеокластов, тем самым останавливая резорбцию кости в этом месте [3].

Очевидно, что сохранение сбалансированного взаимодействия RANK, RANKL и OPG имеет особое зна чение для остеокластогенеза и поддержания физиологического ремоделирования костной ткани. Предполагается, что нарушение данного взаимодействия имеет место при ОП [3].

В условиях физиологической нормы процесс ремоделирования костной ткани не должен приводить к чистой потере кости. Однако после достижения максимальной массы кости (возраст около 18-25 лет) происходят физиологические изменения, приводящие к резорбции костной ткани, превышающей ее образование (возраст около 40 лет). Во время перименопаузы и в течение 5-7 лет после менопаузы у женщин может наблюдаться ускоренная потеря костной массы из-за снижения содержания циркулирующих эстрогенов, которое приводит к увеличению активности остеокластов и повышенной резорбции кости. У пожилых людей потеря костной массы со скоростью примерно 0,5-1\% в год вызвана сочетанием ускоренной резорбции кости и сниженной скорости образования кости. Предпо лагается, что снижение поглощения и абсорбции кальция, снижение концентрации половых гормонов и нарушение функции остеобластов являются основными причинами потери костной массы у пожилых людей $[3,12]$.

ЛС способны приводить к потере костной массы у пациентов любого возраста, вмешиваясь в различные этапы процесса ремоделирования кости. Наиболее распространенный механизм предполагает стимуляцию созревания остеокластов и их функции, что приводит к ускоренной резорбции кости. Некоторые препараты увеличивают резорбцию кости за счет снижения выработки половых гормонов. Снижение активности остеобластов, усиление резорбции костей и нарушение минерализации костей также могут быть вызваны медикаментозным лечением [13,14].

Важную роль в процессе минерализации костной ткани играет витамин D, необходимый для обеспечения надлежащего всасывания в кишечнике кальция который, в свою очередь, и является звеном, необходимым для правильной минерализации кости. Эндогенное образование холекальциферола (витамина D) при воздействии ультрафиолетового света В является основным источником витамина $\mathrm{D}$ в организме человека. Активация холекальциферола происходит в печени и почках. Синтез может быть уменьшен с помощью факторов, которые препятствуют проникновению ультрафиолетового излучения в кожу или нарушают различные этапы его преобразования. Диета служит вторичным источником витамина $\mathrm{D}$, хотя продуктов, которые имеют естественное высокое содержание витамина $\mathrm{D}$, достаточно мало. Сниженные запасы витамина D в организме могут привести к недостаточному усвоению кальция и снижению концентрации его в сыворотке, что может вызвать развитие вторичного гиперпаратиреоза. Гормон паращитовидной железы уменьшает экскрецию кальция почками и увеличивает резорбцию кости, чтобы мобилизовать запасы минерала, увеличивая, таким образом, концентрацию кальция в сыворотке. Потеря костной массы и плохая минерализация костей - последствия дефицита витамина D. Дефицит витамина D также ассоциирован с мышечной слабостью и повышенным риском падений. Некоторые ЛС участвуют в развитии тяжелой недостаточности витамина D, нарушая метаболизм витамина, сопровождающийся развитием гипокальциемии [15].

Менее распространенными механизмами являются гипофосфатемия и прямое влияние на минерализацию костей [16].

\section{Факторы риска лекарственно-индуцированного остеопороза}

Многие пациенты с лекарственно-индуцированным ОП имеют так называемые факторы риска, увеличивающие вероятность потери костной массы и развития переломов. К факторам развития ОП относятся низкое потребление кальция, употребление алкоголя (>2 порций в сутки), курение и недостаточная физическая активность, а также пожилой возраст, женский пол, анамнез, отягощенный падениями и/или переломами, 
сниженные масса тела или индекс массы тела, постменопаузальный статус, отягощенный семейный анамнез в отношении переломов $[3,12,13]$. Некоторые из этих факторов риска включены в модель оценки риска переломов ВО3 (Fracture Risk Assessment Tool, FRAX) [17].

Известно, что некоторые заболевания (хроническая болезнь печени или почек, хроническая обструктивная болезнь легких, депрессия, сахарный диабет 1 и 2-го типов, тиреотоксикоз, гипогонадизм, воспалительные заболевания кишечника, рассеянный склероз, ревматоидный артрит, трансплантация органов, дефицит витамина D), а также ЛС, используемые при их лечении, оказывают неблагоприятное воздействие на костную ткань $[3,12,13]$. При этом зачастую отделить влияние ЛС на ремоделирование кости от влияния самого заболевания бывает трудно.

\section{Клиническая картина и дифференциальная диагностика}

Клиническая картина ОП, индуцированного ЛС, как правило, не отличается от таковой ОП, вызванного другими причинами $[3,12,17]$. Подозрение на лекарственно-индуцированный ОП выше, когда заболевание выявляют у лиц, не имеющих факторов риска ОП (например, мужчины в возрасте до 50 лет или женщины в пременопаузе) [3]. Тем не менее, ЛС следует подозревать в качестве возможной причины ОП у любого пациента, получающего препараты, ассоциированные с потерей костной массы и переломами. Другие причины вторичного ОП, которые следует учитывать, включают в себя следующие [3,18]: злоупотребление алкоголем, генетические расстройства, гипогонадальные состояния, вирус иммунодефицита человека, эндокринные, желудочно-кишечные и гематологические заболевания.

В настоящее время нет единого мнения относительно того, что представляет собой адекватный и экономически эффективный план обследования пациентов с подозрением на вторичный ОП. Так, в дополнение к тщательному сбору анамнеза и физическому обследованию рекомендуют проводить полный общий (клинический) анализ крови, определение активности печеночных ферментов, содержания фосфора, общего белка, альбумина, креатинина, щелочной фосфатазы, электролитов, тиреотропного гормона (ТТГ), паратиреоидного гормона, общего тестостерона и гонадотропина, сывороточного 25(OH)-витамина D, а также сывороточного кальция и кальция в моче $[12,19]$. Также в зависимости от предполагаемой причины ОП могут потребоваться дополнительные лабораторные анализы.

Измерение МПК рекомендуется всем пациентам, принимающим ЛС (например, глюкокортикостероиды в дозе 25 мг в пересчете на преднизолон в течение $>3$ месяцев), которые вызывают снижение костной массы.

\section{Ведение пациентов с лекарственно-индуцированным остеопорозом}

Тактика ведения больных с лекарственно-индуцированным ОП аналогична таковой при лечении первичного
ОП. Одним из важнейших условий эффективной терапии является прекращение приема ЛС, оказывающего негативное влияние на костную ткань, или уменьшение его дозы. Следует придерживаться существующих рекомендаций по предотвращению и лечению лекарственно-индуцированного ОП, развивающегося при приеме тех или иных ЛС (например, глюкокортикостероидов) [20]. При отсутствии конкретных рекомендаций большинство экспертов сходятся во мнении, что бисфосфонаты и другие препараты, рекомендованные для лечения первичного ОП, являются приемлемыми вариантами лечения и лекарственно-индуцированного ОП $[3,20]$. Однако необходимо помнить, что существующие критерии начала терапии ОП не учитывают дополнительные риски, связанные с использованием препаратов, предрасполагающих к его развитию. У пациентов с риском развития лекарственно-индуцированного ОП профилактическое лечение с использованием бисфосфонатов следует рассматривать при величине Т-критерия ниже -1 в позвоночнике или бедре $[12,20]$.

Алендронат, ризедронат, золендроновая кислота и терипаратид одобрены для лечения ОП, вызванного глюкокортикостероидами. Причем терипартид, повидимому, является наиболее эффективным из этих ЛС $[1,21,22]$. Деносумаб не одобрен для терапии стероидного ОП, но его прием ассоциировался со снижением риска переломов у женщин, получающих терапию ингибиторами ароматазы по поводу рака молочной железы, и у мужчин с раком предстательной железы, получающих гормон-депривационную терапию. Препа рат рекомендуется применять для предупреждения низкотравматических переломов и повышения МПК у данной когорты пациентов $[1,23]$.

Другие препараты, которые могут быть рассмотрены для лечения ОП, вызванного ЛС, включают в себя кальцитонин и ралоксифен у женщин (селективный модулятор эстрогенных рецепторов, СМЭР). Приме нение ралоксифена в течение 3-х лет ассоциировалось со снижением риска переломов тел позвонков у пациентов с переломом тела позвонка в анамнезе на $30 \%$ и снижением риска у пациентов без переломов в анамнезе на $55 \%[24,25]$.

Мониторирование уровней витамина $\mathrm{D}$ и кальция и физическая активность - важные компоненты общей терапевтической стратегии и обязательные условия успешной терапии ОП, индуцированного ЛС [3].

\section{Профилактика}

Важнейшими мерами профилактики лекарственноиндуцированного ОП и переломов являются [3]:

- выявление и оценка других возможных факторов риска потери костной массы и развития переломов у лиц, использующих потенциально опасные в отношении костной ткани ЛС;

- сведение к минимуму воздействия потенциально опасных в отношении ОП и переломов ЛС: использование самой низкой дозы и, по возможности, наиболее короткого курса терапии; 
- замена, по возможности, потенциально опасных ЛС на аналогичные, но с меньшим риском отрицательного воздействия на костную ткань, выбор наиболее безопасного способа введения ЛС.

Так, ингаляционные глюкокортикостероиды в низких и средних дозах, в отличие от ингаляционных, не вызывают заметной потери костной массы и предпочтительны для лечения хронической обструктивной болезни легких и бронхиальной астмы [26]. У пациентов, перенесших трансплантацию органа, можно рассмотреть применение таких иммуномодуляторов, как рапамицин, такролимус или микофенолат мофетил, которые с меньшей вероятностью вызывают ОП, чем циклоспорин [3]. Современные противоэпилептические препараты, такие как ламотриджин, топирамат и леветирацетам, вызывают ОП реже, чем фенитоин, карбамазепин или фенобарбитал [3]. Было показано, что ритонавир - ингибитор протеазы, который часто используется для усиления антиретровирусной терапии, ингибирует созревание и функцию остеокластов, способствуя снижению резорбции кости. Ралтегравир или абакавир могут иметь преимущества перед тенофовира дизопроксилом у пациентов с высоким риском остеопении или ОП [27-29]. По сравнению с варфарином прием ривароксабана и апиксабана ассоциирован со значительно более низким риском развития ОП и переломов, поэтому они предпочтительны к назначению при наличии соответствующих показаний [30,31].

Рассмотрение альтернативных вариантов лечения, снижающих риск развития ОП и переломов, особенно важно у пациентов с множеством основных факторов риска потери костной массы и переломов. В свою очередь, прекращение негативного влияния на костную ткань “модифицируемых" факторов риска ОП, имеет важное значение для снижения риска ОП, индуцированного ЛС. Прекращение курения, ограничение потребления алкоголя и кофеина, регулярные физические упражнения, восстановление и поддержание адекватных уровней кальция и витамина D также необходимы для эффективной профилактики и лечения ОП, индуцированного ЛС [1,3].

\section{Заключение}

Проблема ОП - одна из самых актуальных глобальных медико-социальных и экономических проблем, стоящих перед мировым здравоохранением. В свою очередь, проблема ОП, развивающегося в результате использования тех или иных ЛС, привлекает к себе все большее внимание клиницистов. Современные демографические тенденции с увеличением продолжительности жизни и старением населения, рост числа сопутствующих заболеваний, проблема полипрагмазии и активное развитие фармацевтического рынка способствуют развитию лекарственно-обусловленных патологических состояний, включая ОП. При этом диагностика ОП, развивающегося под действием ЛС, зачастую осуществляется достаточно поздно, т.е. после возниквноения первого низкоэнергетического перелома.
Лекарственно-индуцированный ОП ассоциирован с приемом системных глюкокортикостероидов, ингибиторов ароматазы, а также ЛС, угнетающих функцию яичников, препаратов андроген-депривационной терапии, гормонов щитовидной железы, антисекреторных препаратов (ИПП и блокаторов $\mathrm{H}_{2}$-гистаминовых рецепторов), психотропных (противоэпилептических ЛС и антидепрессантов) и сахароснижающих ЛС (тиазолидиндионов), иммуномодуляторов и препаратов, использующихся в кардиологической практике (антикоагулянтов и петлевых диуретиков).

Повышенная осведомленность о ЛС, обладающих негативным влиянием на костную ткань, будет способствовать оптимизации терапии и более частому назначению альтернативных менее опасных препаратов, а также применению адекватных профилактических мер, направленных на предупреждение и снижение риска развития ОП и переломов.

\section{Конфликт интересов: нет.}

1. Российская ассоциация эндокринологов при участии: Российская ассоциация остеопороза, Российская ассоциация ревматологов, Ассоциация травматологов-ортопедов России, Российская ассоциация по менопаузе, Ассоциация гинекологов-эндокринологов. Остеопороз. Клинические рекомендации. М., 2016.

2. Здоровье скелета: проблемы и пути решения. Глобальный план изменения ситуации. Available at: http://share.iofbonehealth.org/WOD/2016/thematicreport/WOD16-report-WEB-RU.pdf.

3. Borgelt LM, Fixen DR. Osteoporosis and osteomalacia. In: Tisdale JE, Miller DA (eds). Drug induced diseases: prevention, detection, and management. 3rd ed.
Bethesda: American Society of Health System Pharmacists; 2018, 1119-33.

4. Hernlund E, Svedbom A, Ivergard M, et al. Osteoporosis in the European Union: medical management, epidemiology and economic burden: A report prepared in collaboration with the International Osteoporosis Foundation (IOF) and the European Federation of Pharmaceutical Industry Associations (EFPIA). Arch Osteoporos 2013;8:136

5. Дедов И.И., Мельниченко Г.А., Белая Ж.Е., Рожинская Л.Я. Остеопороз от редкого симптома эндокринных болезней до безмолвной эпидемии 20-2 века. Проблемы эндокринологии 2011;57:35-45 [Dedov II, Melnichenko GA, Belaya ZhE, Rozhinskaya LYa. Osteoporosis: from a rare symptom of endocrine diseases to the tacit epidemic of XX-XXI centuries. Problemy ehndokrinologii 2011;57:35-45 (In Russ.)].

6. Меньшикова Л.В., Храмцова Н.А., Ершова О.Б. Ближайшие и отдаленные исходы переломов проксимального отдела бедра у лиц пожилого возраста и их медико-социальные последствия. Остеопороз и остеопатии 2002;1:8-11 [Menshikova LV, Khramtsova NA, Ershova OB. Short-term and long-term outcomes of hip fracture in the elderly patients and their medical and social consequences. Osteoporoz i osteopatii 2002;1:8-11 (In Russ.)]

7. O'Connel MB, Borgelt MB, Bowles SK, Vondracek SF. Drug-induced osteoporosis in the older adult. Aging Health 2010;6(4):501-18.

8. Panday K, Gona A, Humphrey MB. Medication-induced osteoporosis: screening and treatment strategies. Ther Adv Musculoskel Dis 2014;6(5):185-202.

9. Mazziotti G, Canalis E, Giustina A. Drug-induced osteoporosis: mechanisms and clinical implications. Amer J Med 2010;123:877-84.

10. Nguyen KD, Bagheri B, Bagheri H. Drug-induced bone loss: a major safety conNguyen KD, Bagheri B, Bagheri H. Drug-induced bone loss:
cern in Europe. Expert Opin Drug Saf 2018;17(10):1005-14.

11. Kelman A, Lane NE. The management of secondary osteoporosis. Best Pract Res Clin Rheumatol 2005;19:1021-37.

12. Cosman F, de Beur SJ, LeBoff MS, et al. Clinician's guide to prevention and treatment of osteoporosis. Osteoporos Int 2014; 25:2359-81.

13. Tannirandorn P, Epstein S. Drug-induced bone loss. Osteoporos Int 2000;11: 637-59.

14. Bode B, Stenlof K, Harris S, et al. Long-term efficacy and safety of canagliflozin over 104 weeks in patients aged 55-80 years with type 2 diabetes. Diabetes Obes Metab 2015;17:294-303.

15. Pasco JA, Henry MJ, Kotowicz MA, et al. Seasonal periodicity of serum vitamin $\mathrm{D}$ and parathyroid hormone, bone resorption, and fractures: the Geelong Osteoporosis Study. J Bone Miner Res 2004;19:752-8.

16. Lips P, van Schoor NM, Bravenboer N. Vitamin D-related disorder. In: Rosen C, ed. Primer on the metabolic bone diseases and disorders of mineral metabolism. 8th ed. Ames, IA: Wiley and Sons Inc. 2013:613-9.

17. FRAX WHO Fracture Risk Assessment Tool. Welcome to FRAX. https:// www.shef.ac.uk/FRAX/ (accessed 2020 March 29).

18. Emkey GR, Epstein S. Secondary osteoporosis: pathophysiology and diagnosis Best Pract Res Clin Endocrinol Metab 2014; 28:911-35.

19. Watts NB, Bilezikian JP, Camacho PM, et al. American Association of Clinical Endocrinologists Medical Guidelines for Clinical Practice for the diagnosis and treatment of postmenopausal osteoporosis: executive summary of recommendatreatment of postmenopausal osteopo
tions. Endocr Pract 2010;16:1016-9. 
20. Grossman JM, Gordon R, Ranganath VK, et al. American College of Rheumatology 2010 recommendations for the prevention and treatment of glucocorticoid-induced osteoporosis. Arthritis Care Res (Hoboken) 2010;62:1515-26.

21. Saag KG, Shane E, Boonen S, et al. Teriparatide or alendronate in glucocorticoid-induced osteoporosis. N Engl J Med 2007;357:2028-39.

22. Gliier CC, Marin F, Ringe JD, et al. Comparative effects of teriparatide and risedronate in glucocorticoid-induced osteoporosis in men: 18-month results of risedronate in glucocorticoid-induced osteoporosis in men:
the Euro GIOPs trial. J Bone Miner Res 2013;28:1355-68

23. Papapoulos S, Chapurlat R, Libanati C, et al. Five years of denosumab exposure in women with postmenopausal osteoporocis: results from the first two years of the FREEDOM extension. J Bone Miner Res 2012;27:694-701.

24. Ettinger B, Black DM, Mitlak BH, et al. Reduction of vertebral fracture risk in postmenopausal women with osteoporosis treated with raloxifene: results from a $3-$ year randomized clinical trial. Multiple Outcomes of Raloxifene Evaluation (MORE) Investigators. JAMA 1999;282:637-45.

25. Chesnut CH 3rd, Silverman S, Andriano K, et al. A randomized trial of nasal spray salmon calcitonin in postmenopausal women with established osteoporosis: the prevent recurrence of osteoporotic fractures study. PROOF Study Group. Am J Med 2000;109:267-76.

26. Buehring B, Viswanathan R, Binkley N, Busse W. Glucocorticoidinduced osteoporosis: an update on effects and management. J Allergy Clin Immunol 2013;132: 1019-30.

27. Brown TT. Challenges in the management of osteoporosis and vitamin D deficiency in HIV infection. Top Antivir Med 2013;21:115-8.

28. Wang MW, Wei S, Faccio R, et al. The HIV protease inhibitor ritonavir blocks osteoclastogenesis and function by impairing RANKL-induced signaling. J Clin Invest 2004;114:206-13.

29. Bernardino JI, Mocroft A, Mallon PW, et al. Bone mineral density and inflammatory and bone biomarkers after darunavir-ritonavir combined with either raltegravir or tenofovir-emtricitabine in antiretroviral-naive adults with HIV-1: a gravir or tenofovir-emtricitabine in antiretroviral-naive adults with HIV-1: a
substudy of the NEAT001/ANRS143 randomised trial. Lancet HIV 2015;2:e46473 .

30. Huang HK, Liu PPS, Hsu JY, et al. Fracture risks among patients with atrial fibrillation receiving different oral anticoagulants: a real-world nationwide cohort study. Europ Heart J 2020;0:1-9. DOI:10.1093/eurheartj/ehz952

31. Huang HK, Liu PPS, Hsu JY, et al. Risk of osteoporosis in patients with atrial fibrillation using non-vitamin $\mathrm{K}$ antagonist oral anticoagulants or warfarin. J Am Heart Assoc 2020;9:e013845. DOI: 10.1161/JAHA.119.013845.

\section{Drug-induced osteoporosis}

\section{O.D. Ostroumova ${ }^{1,2}$, I.V. Goloborodova ${ }^{3}$}

${ }^{1}$ Russian Medical Academy of Continuous Professional Education, ${ }^{2}$ Sechenov First Moscow State Medical University, ${ }^{3}$ Yevdokimov Moscow State University of Medicine and Dentistry, Moscow, Russia

Osteoporosis is characterized by a decrease in bone mass, changes in the microarchitecture of bone tissue, and develop- ment of fractures with minimal trauma. Primary osteoporosis is associated with aging and decreased gonadal function, whereas secondary osteoporosis can be induced by various medications, including hormones (systemic glucocorticosteroids, aromatase inhibitors, depot-medroxyprogesterone, gonadotropin-releasing hormone agonists, levothyroxine), antisecretory (proton pump inhibitors, $\mathrm{H}_{2}$-histamine receptor antagonists), psychotropic (antiepileptic drugs, antidepressants), hypoglycemic (thiazolidinediones) agents, calcineurin inhibitors, antiretroviral drugs, anticoagulants, certain chemotherapeutic agents, loop diuretics. Medicines can lead to bone loss by interfering directly with the various stages of bone remodeling or affecting the levels of sex hormones and calcium-phosphorus metabolism. Risk factors for drug-induced osteoporosis include low calcium intake, alcohol consumption, smoking, inadequate physical activity, older age, female gender, history of weight loss and/or fractures, lower weight or body mass index, postmenopause, family history of fractures. Prevention of drug-induced secondary osteoporosis involves the discontinuation of drugs inducing bone loss. If impossible, these medications should be used in a lower dose for a shorter time in combination with corrective therapy.

Key words. Osteoporosis, drug-induced osteoporosis, bone loss, osteoporotic fracture, drug-induced bone loss, adverse drug reaction.

Conflict of interest: none declared.

Correspondence to: Olga D. Ostroumova. Russian Medical Academy of Continuous Professional Education Russia, Barrikadnaya str., 2/1, Moscow, 125993, Russia. ostroumova.olga@mail.ru.

To cite: Ostroumova OD, Goloborodova IV. Drug-induced osteoporosis. Klinicheskaya farmakologiya i terapiya = Clin Pharmacol Ther 2020;29(3):73-79. DOI 10.32756/0869-54902020-3-73-79. 